\title{
Cortactin promotes colon cancer progression by regulating ERK pathway
}

\author{
QING-FENG NI, JIA-WEI YU, FEI QIAN, NAI-ZHI SUN, JIAN-JIA XIAO and JIAN-WEI ZHU \\ Department of General Surgery, Affiliated Hospital of Nantong University, Nantong, Jiangsu 226001, P.R. China
}

Received April 27, 2015; Accepted June 3, 2015

DOI: 10.3892/ijo.2015.3072

\begin{abstract}
Cortactin is upregulated in various cancers including breast cancer, head and neck squamous cell carcinoma and gastric cancer. However, the role of cortactin in the pathogenesis of colon cancer remains unclear. mRNA expression of cortactin in colon cancer samples and cell lines was detected by quantitative real-time PCR (qRT-PCR), while protein expression of cortactin in colon cancer tissues and adjacent non-cancer tissues was assessed by immunohistochemistry. The role of cortactin in regulation of the proliferation of colon cancer derived cells were investigated both in vitro and in vivo. In the total of 60 paired colon cancer specimens, compared with the adjacent non-cancer tissues, the expression of cortactin mRNA was upregulated in 45 (75.0\%). Immunohistochemical analysis showed significantly increased cortactin expression in colon cancer $(42 / 60,70.0 \%)$ compared to control tissues $(18 / 60,30.0 \%)$. Overexpression of cortactin promoted HCT116 cellular colony formation and tumor growth. Conversely, cortactin knockdown inhibited these effects in SW480 cells. Mechanistic analyses indicated that cortactin was able to activate the EGFR-ERK signaling pathway. Additionally, cortactin expression was associated with tumor size, tumor stages and lymphatic invasion, increased cortactin expression predicts poor prognosis in patients with colon cancer. In summary, cortactin demonstrated the promotive effect in human colon cancer cell growth and tumorigenicity. These results indicated that cortactin may serve as an effective target for gene therapy.
\end{abstract}

\section{Introduction}

Colon cancer is the most common gastrointestinal cancer worldwide, with the incidence increasing in most countries over the past 20 years (1). Colon cancer is often diagnosed at an advanced stage, leading to a poor prognosis (2-5). As the

Correspondence to: Dr Jian-Wei Zhu, Department of General Surgery, Affiliated Hospital of Nantong University, 20 Xisi Road, Nantong, Jiangsu 226001, P.R. China

E-mail: usazhujianwei@aliyun.com

Key words: cortatin, colon cancer, proliferation, EGFR-ERK signaling pathway current clinical procedures utilized for disease diagnosis are invasive, unpleasant, and inconvenient, the development of simple blood tests that can be used for early detection would be beneficial for ultimately controlling and preventing colon cancer $(2,4,5)$. Serum tumor markers, such as carcinoembryonic antigen (CEA) and carbohydrate antigen 199 (CA19-9), greatly improve diagnosis. However, their application is limited to surveillance postsurgery, and they are not suitable for the early detection of colon cancer, as their sensitivity and specificity are very low (6-8). Therefore, it is vital to identify new clues to understand the pathogenesis of colon cancer and to explore effective therapeutic strategies.

Cortactin protein was first identified in chicken cells transformed by the src oncogene. Human cortactin, encoded by the CTTN/EMS1 gene, is a v-Src substrate localized with cortical actin at the plasma membrane (9), cortactin is enriched in cortical structures such as membrane ruffles and lamellipodia, and plays key roles in the microfilament-membrane interactions as well in transducing signals from the cell surface to the cytoskeleton $(10,11)$. Cortactin overexpression results from the 11q11.3 chromosomal region amplification in various cancers, such as head and neck squamous carcinoma, hepatocellular carcinoma, breast and bladder cancer, and correlates with poor patient prognosis and decreased survival (12-15). Clark et al demonstrated that cortactin expression levels in HNSCC cells correlate with the formation of invadopodia and associated with the invasive potential of tumor cells (16). In addition, Wei et al revealed that cortactin overexpression promoted EGFR expression and confers a more malignant phenotype to gastric cancer cells (17).

Despite the many previous studies that were carried out, cotactin expression in colon cncer is still not completely known. Therefore, the present study examined the role of cortactin in colon cancer development, and our results indicated that cortactin is markedly upregulated in colon cancer and can significantly promote colon cancer cell proliferation both in vitro and in vivo.

These data may suggest that cortactin acts as a promoter of colon cancer cell growth and may constitute a potential therapeutic target in colon cancer.

\section{Materials and methods}

Tissue specimens. To examine the expression of cortactin in human colon cancers, sixty surgical resected colon cancers 
and adjacent non-tumor specimens were obtained from Department of General Surgery, Affiliated Hospital of Nantong University. Both colon cancers and adjacent non-tumor tissues were confirmed by pathological examination, and immediately stored in liquid nitrogen after surgery. The study protocols for the investigations involving human tissues were approved by the Ethics Committee of Nantong University.

Colon cancer cell lines. The human colon cancer cell lines (HCT116, HT29, DLD1, LS180, SW480 and SW620) and normal human colon epithelial cells (NCM460) were obtained from American Type Culture Collection (Manassas, VA, USA). HCT116 cells were cultured in McCoy's 5A medium supplemented with $10 \%$ fetal bovine serum, all the other cell lines were cultured in DMEM medium supplemented with $10 \%$ fetal bovine serum. The cell lines were propagated in a $37^{\circ} \mathrm{C}$-humidified incubator with $5 \% \mathrm{CO}_{2}$.

Quantitative real-time PCR. Total RNA from tissue samples and cultured cells was extracted using TRIzol reagent (Invitrogen, Carlsbad, CA, USA) and then reverse transcribed into cDNA using the PrimeScript RT Reagent kit (Takara, Japan). Quantitative real-time PCR (qRT-PCR) assays were carried out to detect mRNA expression using SYBR Premix Ex Taq (Takara, Japan) according to the manufacturer's instructions. Cortactin primers used in qRT-PCR were as follows: forward primer, 5'-TCCCATGGCTATGGAGGGAA-3'; reverse primer, 5'-CGACTGATATTCGTGGCCGA-3'. $\beta$-actin was used as an internal control and amplified with forward primer, 5'-AGAGC CTCGCCTTTGCCGATCC-3'; and reverse primer, 5'-CTGGG CCTCGTCGCCCACATA-3'.

Immunohistochemical staining. All tissues were paraffinembedded and were obtained from the Department of Pathology, Affiliated Hospital of Nantong University. The paraffin-embedded tissues were cut into $4-\mu \mathrm{m}$ sections, and then incubated with rabbit anti-cortactin polyclonal antibody (dilution 1:200; Abcam, Cambridge, UK) at $4^{\circ} \mathrm{C}$ overnight, SP-9000 Histostain ${ }^{\mathrm{TM}}$ Plus kits (ZSGB-BIO) were used according to the manufacturer's protocol. Tumor slices were examined in a blinded manner. Ten fields were selected for examination of the cell-staining intensity and proportion of positive cells. Immunohistochemical staining was assessed according to the immunoreactive score (IRS) that evaluated the staining intensity and the proportion of positive cells. The staining intensity was graded as 0 (no staining), 1 (weak), 2 (moderate) and 3 (strong). The proportion of positive cells was scored as 0 (negative), $1(<10 \%), 2(10-50 \%)$ and $3(>50 \%)$. Both of the scores were multiplied and the IRS was determined: values $\geq 3$ were defined as cytoplasmic expression positive, and values $<3$ were regarded as negative.

shRNA preparation. SiRNA against cortactin was designed according to the Invitrogen Co. and chemically synthesized by Shanghai GenePharma Co. (Shanghai, China). The sequence of siRNA-410 was: CCUUAAGGAGAAGGAAC UUdTdT (sense) and AAGUUCCUUCUCCUUAAGGdTdT (antisense). Negative control (NC) siRNA synthesized by Shanghai GenePharma Co. was used as a control. The sequence of si-NC was as follows: UUCUCCGAACGUGUCACGUTT (sense) and ACGUGACACGUUCGGAGAATT (antisense). ShRNA duplexes against cortactin was designed according to the Invitrogen Co. and synthesized by GenePharma Co. The sequences were incorporated into the Vector p-SUPER (OligoEngine, USA) to generate p-SUPER-shRNA-cortactin. The sequence of shRNA-410 was as follows: GATCCCCCCTT AAGGAGAAGGAACTTTTCAAGAGAAAGTTCCTTCTC CTTAAGGTTTTTGGAAA (sense) and AGCTTTTCCAAA AACCTTAAGGAGAAGGAACTTTCTCTTGAAAAGTTC CTTCTCCTTAAGGGGG (antisense). The sequence of sh-NC was as follows: GATCCCCTTCT CCGAACGTGTCACGTTT CAAGAGAACGTGACACGTTCGGAGAATTTTTGGAAA (sense) and AGCTTTTCCAAAAATTCTCCGAACGTGT CACGTTCTCTTGAAACGTGACACGTTCGGAGAAGGG (antisense). The constructs were verified by sequencing.

Construction of cortactin expression vector. The cortactin open reading frame (ORF) was amplified from the human colon cDNA library (Genbank: NM_001184740.1) using Prime Star PCR and constructed into the expression vector pcDNA1.1B to generate pcDNA1.1B-cortactin. The sequence of the forward primer was as follows: 5'-EcoRI-AGAGAATTCATGTGGA AAGCTTCAGCA-3' and reverse primer' 5'-BamHI-AGA GGATCCTCACGGGCACTCCGGGAC-3'. The construct was verified by sequencing.

Cell transfection. Both the shRNA and cortactin expression vector were transfected using Lipofectamine 2000 (Invitrogen) according to the manufacturer's instructions.

Cell proliferation assay. Cells were seeded at a density of 2,000-5,000 cells/well in $100 \mu \mathrm{l}$ complete medium in 96-well plates. The Cell Counting Kit-8 (Dojindo Labs) was used to measure cell viability according to the manufacturer's instructions. Each experiment was repeated at least 3 times.

Colony formation assay. To examine the effect of upregulated or downregulated cortactin expression on proliferation of colon cancer cell lines, cells transfected with different plasmids were used for the colony formation assay. Each type of cell was seeded into $10-\mathrm{cm}$ plates $(50,000$ cells/well) and cultured for 3 weeks in medium containing $1,000 \mu \mathrm{g} / \mathrm{ml} \mathrm{G} 418$. These cultures were stained with $0.4 \%$ crystal violet. Clones $>2 \mathrm{~mm}$ were counted and the number of clones per well was averaged from three wells for each experiment. Each experiment was repeated at least 3 times.

Soft agar colony formation assay. Cells transfected with different plasmids were suspended in $0.5 \mathrm{ml} 1 \%$ low melting point agarose with complete culture medium, and then layered on top of $0.5 \mathrm{ml} 2 \%$ low melting agarose in 24 -well plates. Cell numbers varied from 2,000 to 5,000 for different cell lines. The plates were incubated in a $5 \% \mathrm{CO}_{2}, 37^{\circ} \mathrm{C}$-humidified incubator for 2 weeks. Colonies in at least 6 random microscopic fields were counted and photographed. All experiments were repeated 3 times.

Tumorigenicity assay in nude mice. Male BALB/c nude mice (3-4-week-old) were purchased from the Department of Laboratory Animal Center, Nantong University. Cells with 
differential cortactin expression were injected subcutaneously into the lateral root of the anterior limb of nude mice (500x $10^{6}$ cells/mouse, 4 mice in each experimental group). Tumor size was measured every third day after injection. Three weeks after injection, the mice were sacrificed and photographed. Tumor weight was then calculated. Care of experimental animals was in accordance with institutional animal care and use committee guidelines.

Western blot analysis. Cell lysates were prepared using cold lysis buffer containing $25 \mathrm{mmol} / \mathrm{l}$ Tris- $\mathrm{Cl}(\mathrm{pH} \mathrm{7.5),} 5 \mathrm{mmol} / \mathrm{l}$ EDTA, $1 \%$ SDS and protease inhibitor cocktail (Sigma, USA). After boiling for $5 \mathrm{~min}$, samples were subjected to electrophoresis in 10\% SDS-PAGE and transferred onto a polyvinylidene difluoride (PVDF) membrane, and blocked for $1 \mathrm{~h}$ at room temperature with $5 \%$ blocking buffer. The membranes were washed three times with $0.1 \%$ Tris-buffered saline with Tween-20 (TBST) and incubated with the primary antibody overnight at $4^{\circ} \mathrm{C}$. The membranes were washed again and then incubated with the secondary antibody at room temperature for $1 \mathrm{~h}$. Primary antibodies used in this study included: rabbit anti-cortactin polyclonal antibody $(1: 1,000$; Abcam), rabbit anti-EGFR polyclonal antibody (1:200; Abcam), rabbit anti-ERK polyclonal antibody (1:1,000; Abcam), rabbit anti-pERK polyclonal antibody (1:500; Abcam), rabbit anticyclin D1 polyclonal antibody (1:1,000; Abcam) and rabbit anti- $\beta$-actin polyclonal antibody (1:1,000; Abcam). Proteins were detected using an ECL Western blotting detection system (Pierce) by enhanced chemiluminescence.

Patient follow-up. The total of 60 patients underwent resection of colon cancer with curative intent at the Department of General Surgery, Affiliated Hospital of Nantong University between March 2009 and February 2010. Curative resection was considered as complete resection of tumor mass, with the tumor margins free, and resection of the regional lymph nodes. Patients with distant metastasis were eliminated from this study. The patients were followed from March 2010 to February 2015. The detailed clinicopathological characteristics of the 60 patients with colon cancer are listed in Table I.

Statistical analysis. Statistical analysis was performed using SPSS 18.0 and Graphpad Prism 5.0 software. Quantitative data were recorded as mean \pm SD. Differences between two groups were assessed by Student's t-test (two-tailed). Categorical data were evaluated by the $\chi^{2}$ test. Survival rates were assessed by the Kaplan-Meier method. The log-rank test was used to compare significance. $\mathrm{P}<0.05$ was considered statistically significant.

\section{Results}

Cortactin expression in colon cancer tissues and colon cancer cell lines. Quantitative real-time PCR was performed to measure the cortactin mRNA expression levels in colon cancer and adjacent non-cancerous tissues from 60 patients. The results indicated that cortactin mRNA was increased in $45(75 \%)$ of 60 colon cancer specimens compared with the matched normal colon tissues (Fig. 1A). We performed immunohistochemistry (IHC) to evaluate cortactin protein

Table I. Expression of cortactin in human colon cancer according to clinicopathological features of patients.

\begin{tabular}{|c|c|c|c|c|}
\hline \multirow[b]{2}{*}{$\begin{array}{l}\text { Clinicopathological } \\
\text { features }\end{array}$} & \multicolumn{2}{|c|}{ Cortactin expression } & \multirow[b]{2}{*}{$\chi^{2}$ value } & \multirow[b]{2}{*}{ P-value } \\
\hline & $\begin{array}{c}\text { Strong }(+) \\
\mathrm{n}=34\end{array}$ & $\begin{array}{c}\text { Weak (-) } \\
n=26\end{array}$ & & \\
\hline
\end{tabular}

Age (years)

$<50$

$\geq 50$

15

19

11

15

0.020

0.889

Gender

Male

Female

18

16

14

Depth

Localized in

11

12

0.005

0.944

subserosa

Beyond subserosa

8

Tumor size

$$
<3
$$

$\geq 3$

8

26

13

13

4.538

0.033

Stage

I/II

III/IV

\section{6} 28

12

14

5.701

0.017

Lymphatic invasion

\begin{tabular}{lrrrr} 
Absent & 5 & 13 & & \\
Present & 29 & 13 & 8.740 & 0.003 \\
\hline
\end{tabular}

Strong (+) cortactin expression, cortactin expression in colon cancers/ cortactin expression in normal tissues $\geq 2$; weak (-) cortactin expression, cortactin expression in colon cancers/cortactin expression in normal tissues $<2$; $\mathrm{P}<0.05$, statistically significant difference.

expression in colon cancer specimens and paired normal colon tissues in the same 60 matched samples. Of these specimens, $42 / 60(70.0 \%)$ of cancerous specimens showed positive staining, whereas $18 / 60(30.0 \%)$ of normal colon tissues showed positive staining (Fig. 1C). Furthermore, we evaluated the expression of cortactin in 6 colon cancer cell lines using quantitative real-time PCR. Cortactin mRNA was significantly increased in all colon cancer derived cell lines (HCT116, HT29, DLD1, LS180, SW480 and SW620) compared with normal human colon epithelial cells (NCM460) (Fig. 1B). In summary, the collective data showed that cortactin is increased in colon cancer.

Correlation of cortactin expression with the patient clinicopathological features. To investigate whether the increased expression of cortactin was associated with the clinicopathological features such as age, gender, depth, tumor size, tumor stages and lymphatic invasion, we classified the patients into two groups on the basis of our qRT-PCR results for cortactin: weak expression (-) and strong expression (+). As shown in Table I, those patients with tumor size $(\geq 3 \mathrm{~cm})$ had a signifi- 
A

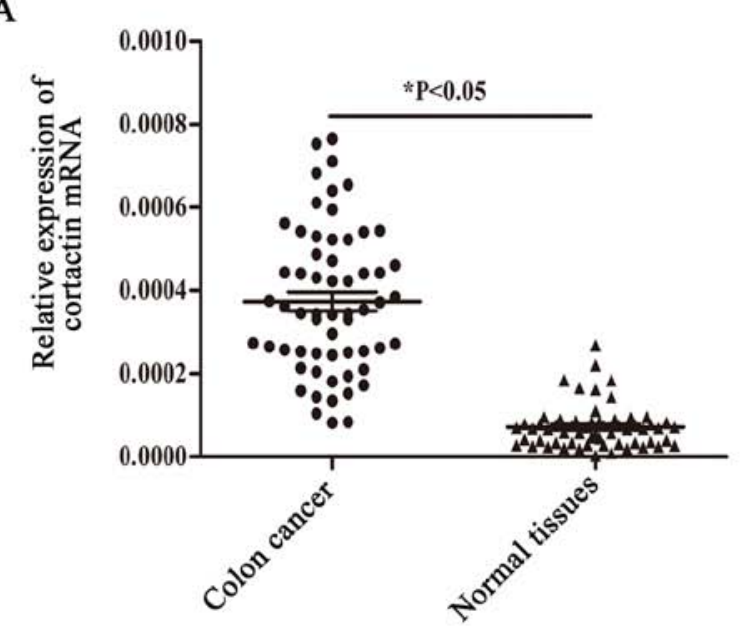

B

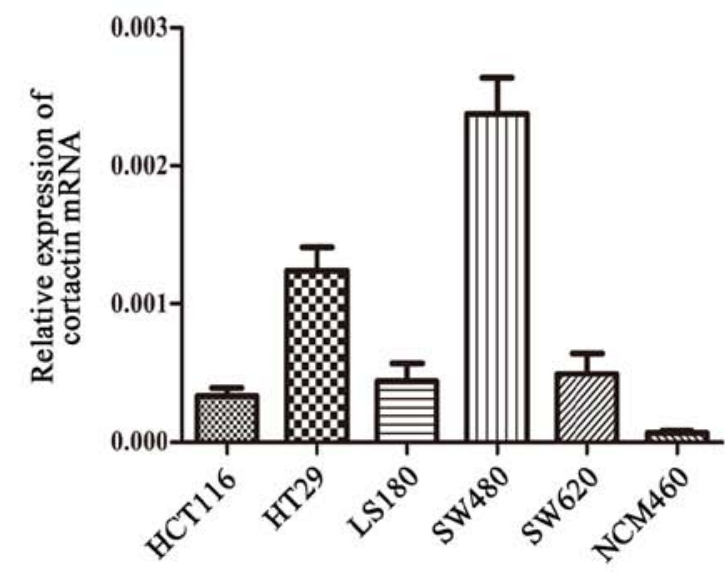

C

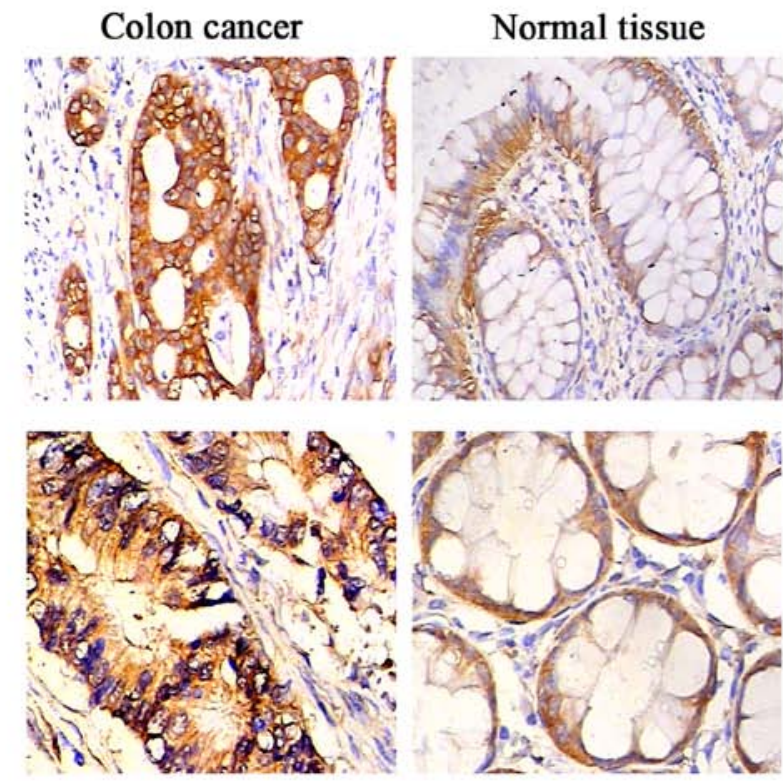

D

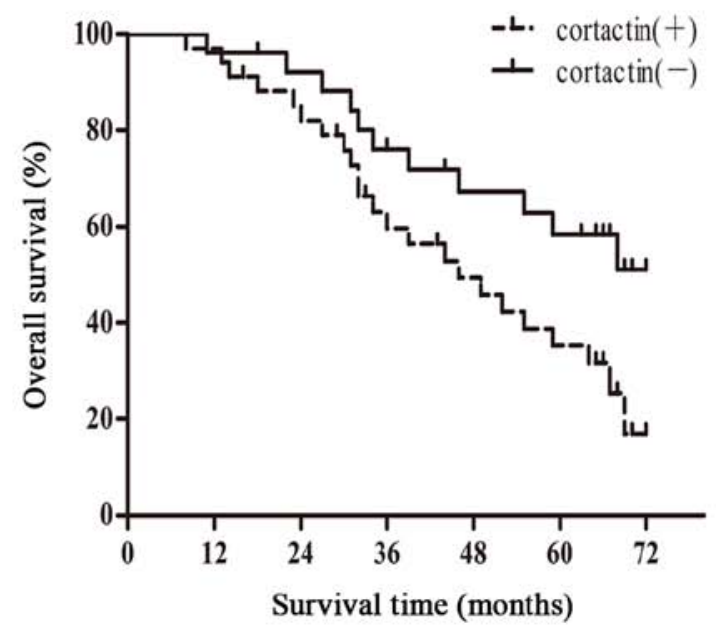

Figure 1. Upregulation of cortactin in clinical specimens and colon cancer derived cell lines. (A) The expression of cortactin mRNA in 60 paired colon cancer specimens by qRT-PCR $(* \mathrm{P}<0.05)$. (B) Cortactin mRNA was upregulated in 6 colon cancer derived cell lines. (C) Representative results of the upregulation of cortactin protein in colon cancer specimens by immunohistochemistry (x400 magnification). (D) Compared with the weak expression (-) cortactin group, the patients' overall survival (OS) was lower in the strong expression $(+)$ cortactin group $\left({ }^{*} \mathrm{P}<0.05\right)$.

cantly higher expression of cortactin compared with those patients with tumor size $(<3 \mathrm{~cm})$. In addition, in stages III/IV, expression of cortactin was dramatically higher than stages I/II. Furthermore, lymphatic invasion was significantly correlated with a strong cortactin expression, whereas no significant difference was observed in terms of the patient age, gender and tumor depth. In summary, cortactin functions as an oncogene accelerator in colon cancer.

Increased expression of cortactin predicts poor prognosis in patients with colon cancer. Out of the 60 cases, there were 52 patients with definite follow-ups. A total of 34 patients died, of whom 23 showed strong expression of cortactin, 11 showed weak expression of cortactin. The 5-year OS rates of patients with strong cortactin expression were $16.9 \%$, which were lower than those of patients with weak cortactin expression (51.1\%) (Fig. 1D).
Overexpression of cortactin promotes proliferation and colony formation in HCT116 cells. To overexpress cortactin, the recombinant pcDNA1.1B-cortactin was transfected into the HCT116 cells. We performed western blotting to evaluate cortactin protein expression in HCT116 cells transfected with pcDNA1.1B-cortactin $48 \mathrm{~h}$ post-transfection. Cortactin protein expression in pcDNA1.1B-cortactin transfected cell was significantly higher than in pcDNA1.1B transfected cell (Fig. 2A). To investigate the promotive effects in pcDNA1.1Bcortactin transfected cell, cellular growth was monitored for 5 days. The pcDNA1.1B-cortactin transfected HCT116 cells showed a significant increase in cellular growth compared with pcDNA1.1B transfected cells (Fig. 2B). HCT116 cells with upregulated cortactin expression were subjected to colony formation assay. As shown in Fig. 2C, overexpression of cortactin in HCT116 cells resulted in significant promotion of colony formation as compared with HCT116 cells transfected 
A

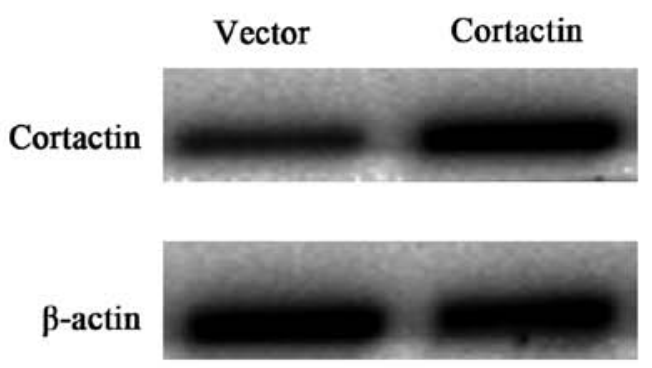

$\mathrm{C}$

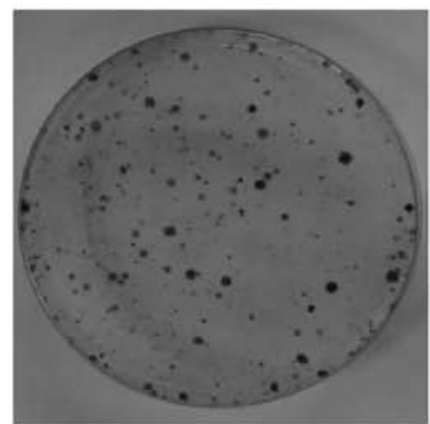

Vector

D

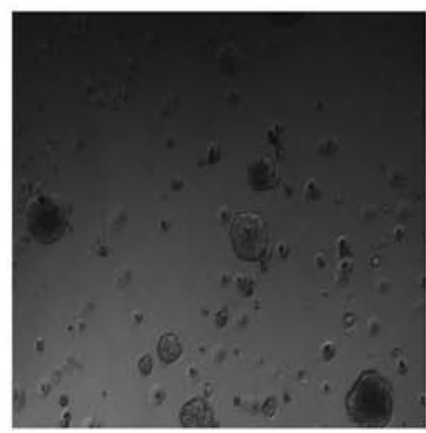

Vector

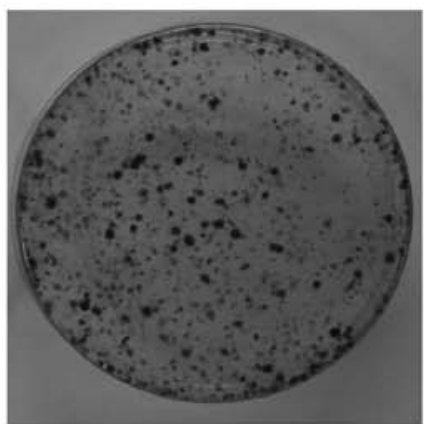

Cortactin

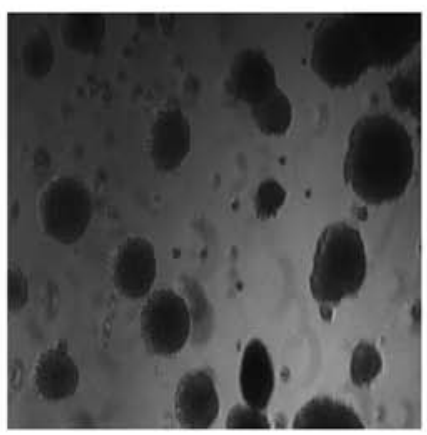

Cortactin
B
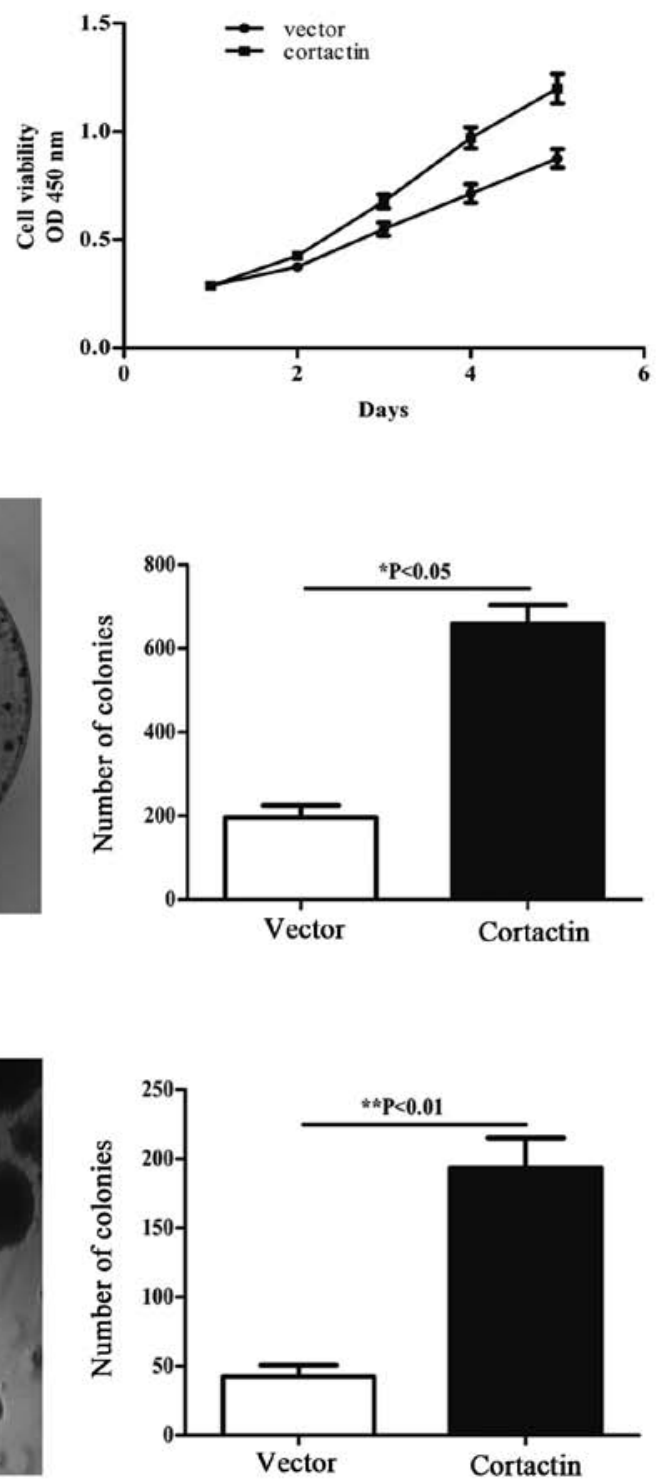

Figure 2. Overexpression of cortactin promotes proliferation and colony formation. (A) HCT116 cells transfected with pcDNA1.1B-cortactin stably increased cortactin expression shown by western blotting. (B) Overexpression of cortactin promotes proliferation of HCT116 cells ( $\mathrm{P}<0.05$ ). (C) Overexpression of cortactin promotes colony formation of HCT116 cells $\left({ }^{*} \mathrm{P}<0.05\right)$. (D) Overexpression of cortactin promotes soft agar colony formation of $\mathrm{HCT} 116$ cells $\left({ }^{* *} \mathrm{P}<0.01\right)$.

with pcDNA1.1B, and the majority of clones were larger than those of control cells. We then used a soft agar assay for colony formation, which is the most stringent assay for detecting the proliferative ability of cells. We observed enhanced formation of colonies in soft agar (Fig. 2D) that had been seeded with HCT116 cells transfected with pcDNA1.1B-cortactin compared with pcDNA1.1B transfected cells.

Knockdown of cortactin inhibits proliferation and colony formation in SW480 cell line. To knockdown cortactin, the recombinant p-SUPER-shRNA-cortactin was transfected into SW480 cells. Western blot analyses were performed to assess the efficiency of cortactin knockdown in SW480 cells transfected with p-SUPER-shRNA-cortactin $48 \mathrm{~h}$ post-transfection. Cortactin protein expression in p-SUPER-shRNA-cortactin transfected cells was significantly lower than that in sh-NC transfected cells (Fig. 3A). The p-SUPER-shRNA-cortactin transfected SW480 cells showed significant suppression of cellular growth compared with sh-NC transfected cells (Fig. 3B). SW480 cells with downregulated cortactin expression were subjected to colony formation assay. As shown in Fig. 3C, decreased expression of cortactin in SW480 cells resulted in significant suppression of colony formation as compared with cells transfected with sh-NC. We also observed inhibited formation of colonies in soft agar (Fig. 3D) when seeded with SW480 cells transfected with p-SUPER-shRNAcortactin compared with sh-NC transfected cells.

Differential expression of cortactin influences tumorigenesis in nude mice. The effects of differential cortactin expression 
A

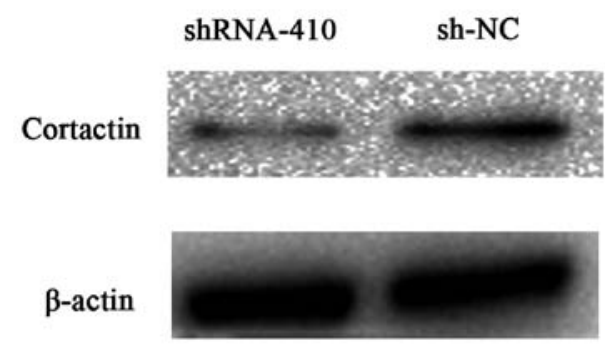

C

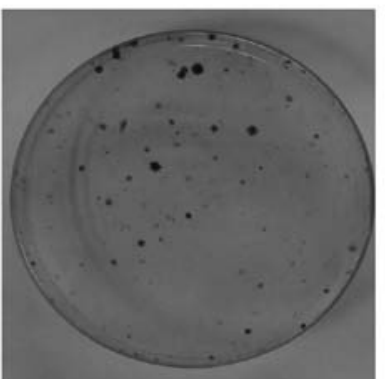

shRNA-410

D

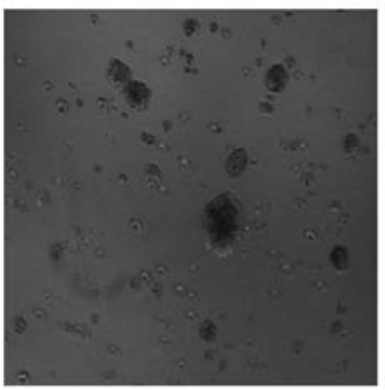

shRNA-410

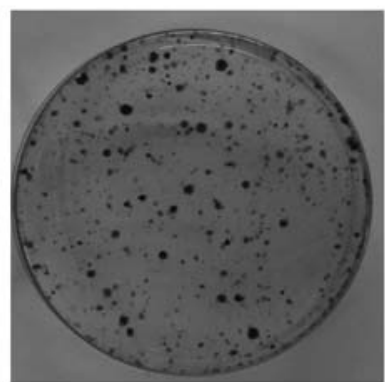

sh-NC

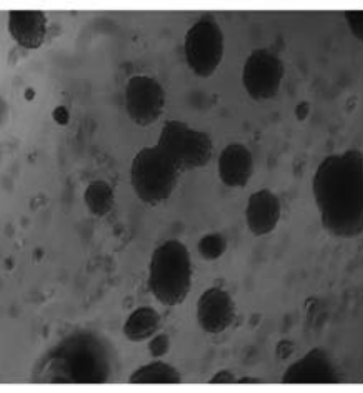

sh-NC
B
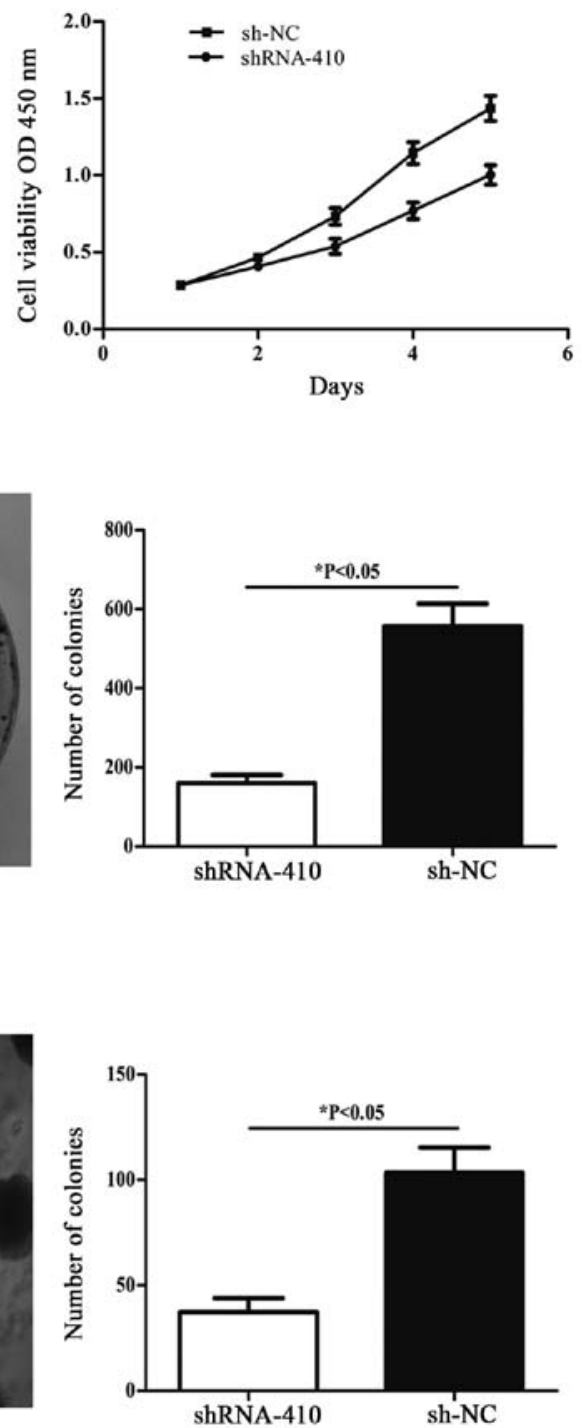

Figure 3. Knockdown of cortactin inhibits proliferation and colony formation. (A) SW480 cells transfected with p-SUPER-shRNA-cortactin stably decreased cortactin expression shown by western blotting. (B) Knockdown of cortactin inhibits proliferation of SW480 cell (*P<0.05). (C) Knockdown of cortactin inhibits colony formation of SW480 cells ( $\mathrm{P}<0.05)$. (D) Knockdown of cortactin inhibits soft agar colony formation of SW480 cells ( $\mathrm{P}<0.05)$.

on the tumorigenic potential of colon cancer cells in vivo were also evaluated. HCT116 cells overexpressing cortactin and SW480 with downregulated cortactin expression were injected subcutaneously into BALB/c nude mice $\left(500 \times 10^{6}\right.$ cells/mouse, 4 mice in each experimental group). Tumor size was measured every third day after injection. After 3 weeks, mice were sacrificed and photographed, and the tumors were removed and weighed. In comparison with the mice injected with HCT116 cells transfected with pcDNA1.1B, the mice injected with HCT116 cells transfected with pcDNA1.1B-cortactin displayed larger tumors during the same time period, and the average tumor volumes and weights were significantly higher than those in the control group (Fig. 4A, C and E). Compared with the mice injected with SW480 cells transfected with p-SUPER-sh-NC, the mice injected with SW480 cells transfected with p-SUPER-shRNA-cortactin showed an obvious decreased capacity for tumorigenesis (Fig. 4B, D and F).
Taken together, these results strongly suggest that cortactin acts as an promotor of tumor cell growth and tumorigenicity in vivo.

Differential expression of cortactin influences the EGFR-ERK signaling pathway related proteins. To study the mechanism by which cortactin enhances the growth of colon cancer, the EGFR, ERK, p-ERK, cyclin D1 protein levels were detected by western blotting. As shown in Fig. 5, the levels of EGFR, p-ERK and cyclin D1 were increased in the pcDNA1.1Bcortactin transfected HCT116 cells compared with the pcDNA1.1B transfected HCT116 cells, and no difference in the tERK protein levels were observed between the two cell groups. The levels of EGFR, p-ERK, cyclin D1 were decreased in the p-SUPER-shRNA-cortactin transfected SW480 cells compared with the sh-NC transfected SW480 cells. Similarly, the expression of tERK was not different between the cell 


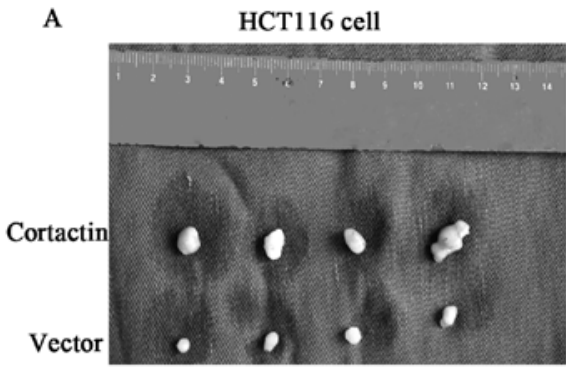

C

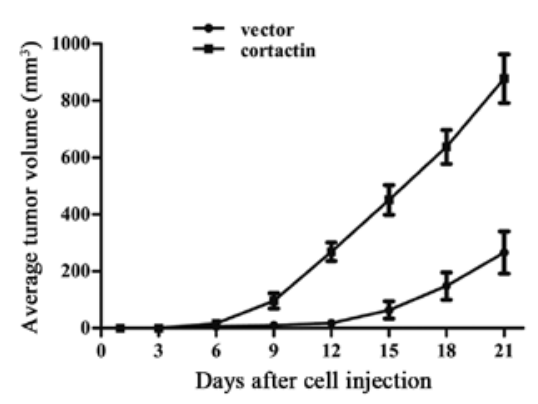

E

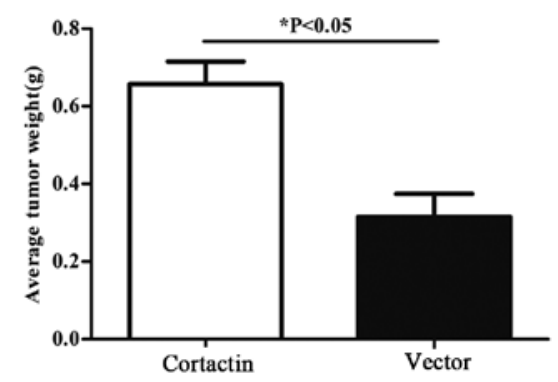

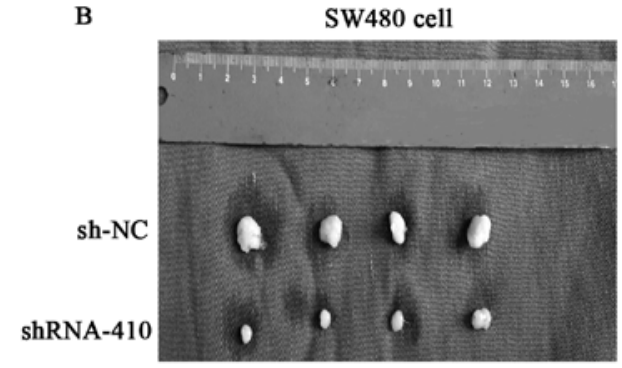

D

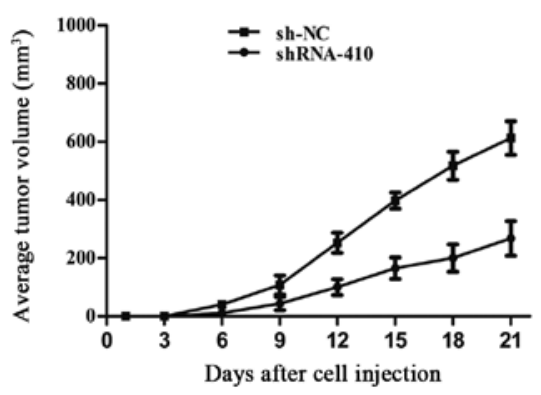

F

SW480 cell

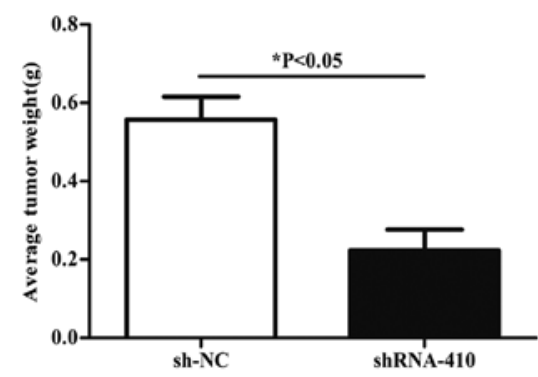

Figure 4. The effect of cortactin expression on tumorigenesis in nude mice. (A and B) Tumors were excised 21 days after injection. (C and D) Tumor volume was measured every third day after injection $(" \mathrm{P}<0.05)$. ( $\mathrm{E}$ and $\mathrm{F})$ The average weight of tumors in each group was assessed $\left({ }^{*} \mathrm{P}<0.05\right)$.
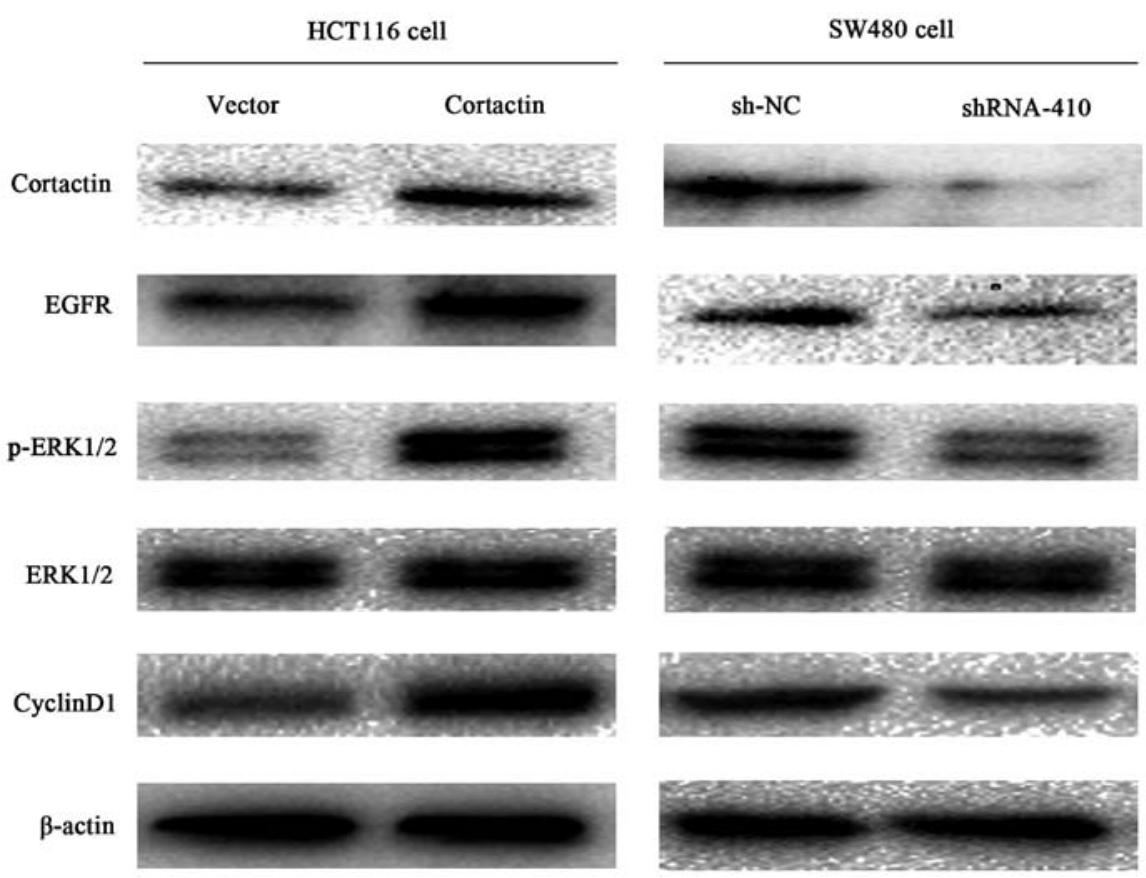

Figure 5. Western blot analysis of EGFR-ERK signaling pathway related proteins showed upregulation of EGFR, p-ERK, cyclin D1 in cortactin-overexpressed HCT116 cells and downregulation of EGFR, pERK, cyclin D1 in cortactin-silenced SW480 cell, tERK protein level was unchanged. 
groups. These results indicate that cortactin overexpression enhances the EGFR expression and in turn constitutively activates the EGFR-ERK signaling pathway.

\section{Discussion}

Recurrence and metastasis remain the major causes of treatment failure and poor prognosis in patients with colon cancer, and it is a multistage process that involves the motility and migration of cells and proliferation in a new site (18). Cortactin is a multidomain protein consisting of an $\mathrm{NH} 2$-terminal acidic region, which binds the actin related protein (Arp)2/3 complex, a repeat region, which associates with filamentous actin, and a $\mathrm{COOH}$-terminal src homology (SH)3 domain, which recruits a variety of cellular proteins (19). One cellular role for cortactin is to bridge the endocytic machinery with components and regulators of the actin cytoskeleton (20). This interaction has recently been shown to participate in receptor-mediated endocytosis (20-22). The gene encoding cortactin, EMS1, localizes to chromosomal locus 11q13, a region commonly amplified in breast cancers and head and neck squamous cell carcinoma $(23,24)$. Since cortactin overexpression increases the motility of fibroblasts and endothelial cells $(25,26)$, enhances bone metastasis of MDA-MB-231 breast cancer cells in a nude mouse model (27) and promotes gastric cancer cell SGC-7901 proliferation (17).

In the present study, we first determined the expression of cortactin in colon cancer, and further investigated its role in colon cancer cell proliferation and tumorigenicity both in vitro and in vivo.

EGFR is frequently implicated in cancer cell proliferation, inhibition of apoptosis and tumor-induced neovascularization via the activation of downstream signaling pathways. EGFR is often overexpressed in various types of tumors, such as salivary gland carcinomas, non-small cell lung cancer, biliary tract cancer, and gastric cancer (28-32). One of the most frequent targets downstream of EGFR and other receptor tyrosine kinases is the extracellular signal-regulated kinase (ERK) kinase signal transduction pathway. This pathway is involved in proliferation, differentiation, apoptosis, and angiogenesis (33). It is constitutively active in a variety of solid tumor models, including lung, pancreas, and breast (33). Elevated levels of activated ERK are frequently seen in carcinoma cell lines $(34,35)$. Activation of the ERK pathway regulates the activity of a number of substrates through phosphorylation (36).

In this study, we first determined the expression of EGFR and ERK in colon cancer cells, it revealed that cortactin enhances EGFR expression and ERK phosphorylation. Sustained ERK activity can upregulate cyclin D1 expression.

In conclusion, our findings show that cortactin is significantly upregulated in colon cancer, while ectopic cortactin expression promotes cell proliferation, colony formation, soft agar colony formation and tumorigenicity. Further investigation revealed that cortactin stimulates the EGFR-ERK signaling pathway. This study is the first to discover that cortactin expression plays an important role in colon cancer progression, but further studies are required to identify the detailed interaction between cortactin and EGFR-ERK signaling pathway in colon cancer, and whether cortactin could act as a therapeutic agent for colon cancer patients should be investigated.

\section{References}

1. Ferlay J, Shin HR, Bray F, Forman D, Mathers C and Parkin DM: Estimates of worldwide burden of cancer in 2008: GLOBOCAN 2008. Int J Cancer 127: 2893-2917, 2010.

2. Fan NJ, Kang R, Ge XY, Li M, Liu Y, Chen HM and Gao CF: Identification alpha-2-HS-glycoprotein precursor and tubulin beta chain as serology diagnosis biomarker of colorectal cancer. Diagn Pathol 9: 53, 2014.

3. Thomson DMP, Krupey J, Freedman SO and Gold P: The radioimmunoassay of circulating carcinoembryonic antigen of the human digestive system. Proc Natl Acad Sci USA 64: 161-167, 1969.

4. Duffy MJ: Role of tumor markers in patients with solid cancers: A critical review. Eur J Intern Med 18: 175-184, 2007.

5. Roulston JE: Limitations of tumour markers in screening. Br J Surg 77: 961-962, 1990.

6. Bagaria B, Sood S, Sharma R and Lalwani S: Comparative study of CEA and CA19-9 in esophageal, gastric and colon cancers individually and in combination (ROC curve analysis). Cancer Biol Med 10: 148-157, 2013.

7. Tan E, Gouvas N, Nicholls RJ, Ziprin P, Xynos E and Tekkis PP: Diagnostic precision of carcinoembryonic antigen in the detection of recurrence of colorectal cancer. Surg Oncol 18: 15-24, 2009.

8. Flamini E, Mercatali L, Nanni O, Calistri D, Nunziatini R, Zoli W, Rosetti P, Gardini N, Lattuneddu A, Verdecchia GM, et al: Free DNA and carcinoembryonic antigen serum levels: An important combination for diagnosis of colorectal cancer. Clin Cancer Res 12: 6985-6988, 2006.

9. Wu H, Reynolds AB, Kanner SB, Vines RR and Parsons JT: Identification and characterization of a novel cytoskeletonassociated pp60src substrate. Mol Cell Biol 11: 5113-5124, 1991.

10. Wu H and Parsons JT: Cortactin, an 80/85-kilodalton pp60src substrate, is a filamentous actin-binding protein enriched in the cell cortex. J Cell Biol 120: 1417-1426, 1993.

11. Weed SA and Parsons JT: Cortactin: Coupling membrane dynamics to cortical actin assembly. Oncogene 20: 6418-6434, 2001.

12. Bringuier PP, Tamimi Y, Schuuring E and Schalken J: Expression of cyclin D1 and EMS1 in bladder tumours; relationship with chromosome 11q13 amplification. Oncogene 12: 1747-1753, 1996.

13. Yuan BZ, Zhou X, Zimonjic DB, Durkin ME and Popescu NC: Amplification and overexpression of the EMS 1 oncogene, a possible prognostic marker, in human hepatocellular carcinoma. J Mol Diagn 5: 48-53, 2003.

14. Rothschild BL, Shim AH, Ammer AG, Kelley LC, Irby KB, Head JA, Chen L, Varella-Garcia M, Sacks PG, Frederick B, et al: Cortactin overexpression regulates actin-related protein $2 / 3$ complex activity, motility, and invasion in carcinomas with chromosome 11q13 amplification. Cancer Res 66: 8017-8025, 2006.

15. Hofman P, Butori C, Havet K, Hofman V, Selva E, Guevara N, Santini J and Van Obberghen-Schilling E: Prognostic significance of cortactin levels in head and neck squamous cell carcinoma: Comparison with epidermal growth factor receptor status. Br J Cancer 98: 956-964, 2008.

16. Clark ES, Whigham AS, Yarbrough WG and Weaver AM: Cortactin is an essential regulator of matrix metalloproteinase secretion and extracellular matrix degradation in invadopodia. Cancer Res 67: 4227-4235, 2007.

17. Wei J, Zhao Z-X, Li Y, Zhou Z-Q and You T-G: Cortactin expression confers a more malignant phenotype to gastric cancer SGC-7901 cells. World J Gastroenterol 20: 3287-3300, 2014.

18. Zhang YY, Chen B and Ding YQ: Metastasis-associated factors facilitating the progression of colorectal cancer. Asian Pac J Cancer Prev 13: 2437-2444, 2012.

19. Daly RJ: Cortactin signalling and dynamic actin networks. Biochem J 382: 13-25, 2004.

20. McNiven MA, Kim L, Krueger EW, Orth JD, Cao H and Wong TW: Regulated interactions between dynamin and the actin-binding protein cortactin modulate cell shape. J Cell Biol 151: 187-198, 2000.

21. Cao H, Orth JD, Chen J, Weller SG, Heuser JE and McNiven MA: Cortactin is a component of clathrin-coated pits and participates in receptor-mediated endocytosis. Mol Cell Biol 23: 2162-2170, 2003. 
22. Engqvist-Goldstein AE, Zhang CX, Carreno S, Barroso C Heuser JE and Drubin DG: RNAi-mediated Hip1R silencing results in stable association between the endocytic machinery and the actin assembly machinery. Mol Biol Cell 15: 1666-1679, 2004.

23. Hui R, Campbell DH, Lee CS, McCaul K, Horsfall DJ, Musgrove EA, Daly RJ, Seshadri R and Sutherland RL: EMS1 amplification can occur independently of CCND1 or INT-2 amplification at 11q13 and may identify different phenotypes in primary breast cancer. Oncogene 15: 1617-1623, 1997.

24. Rodrigo JP, García LA, Ramos S, Lazo PS and Suárez C: EMS1 gene amplification correlates with poor prognosis in squamous cell carcinomas of the head and neck. Clin Cancer Res 6: 3177-3182, 2000.

25. Patel AS, Schechter GL, Wasilenko WJ and Somers KD: Overexpression of EMS1/cortactin in NIH3T3 fibroblasts causes increased cell motility and invasion in vitro. Oncogene 16: 3227-3232, 1998.

26. Huang C, Liu J, Haudenschild CC and Zhan X: The role of tyrosine phosphorylation of cortactin in the locomotion of endothelial cells. J Biol Chem 273: 25770-25776, 1998.

27. Li Y, Tondravi M,Liu J, Smith E, Haudenschild CC, Kaczmarek M and Zhan X: Cortactin potentiates bone metastasis of breast cancer cells. Cancer Res 61: 6906-6911, 2001.

28. Clauditz TS, Gontarewicz A, Lebok P, Tsourlakis MC, Grob TJ Münscher A, Sauter G, Bokemeyer C, Knecht R and Wilczak W: Epidermal growth factor receptor (EGFR) in salivary gland carcinomas: Potentials as therapeutic target. Oral Oncol 48: 991-996, 2012.

29. Gazdar AF: Epidermal growth factor receptor inhibition in lung cancer: The evolving role of individualized therapy. Cancer Metastasis Rev 29: 37-48, 2010.
30. Pirker R, Pereira JR, von Pawel J, Krzakowski M, Ramlau R, Park K, de Marinis F, Eberhardt WE, Paz-Ares L, Störkel S, et al: EGFR expression as a predictor of survival for first-line chemotherapy plus cetuximab in patients with advanced non-small-cell lung cancer: Analysis of data from the phase 3 FLEX study. Lancet Oncol 13: 33-42, 2012.

31. Harder J, Waiz O, Otto F, Geissler M, Olschewski M, Weinhold B, Blum HE, Schmitt-Graeff A and Opitz OG: EGFR and HER2 expression in advanced biliary tract cancer. World J Gastroenterol 15: 4511-4517, 2009.

32. Galizia G, Lieto E, Orditura M, Castellano P, Mura AL, Imperatore V, Pinto M, Zamboli A, De Vita F and Ferraraccio F: Epidermal growth factor receptor (EGFR) expression is associated with a worse prognosis in gastric cancer patients undergoing curative surgery. World J Surg 31: 1458-1468, 2007.

33. Sebolt-Leopold JS and Herrera R: Targeting the mitogen-activated protein kinase cascade to treat cancer. Nat Rev Cancer 4: 937-947, 2004.

34. Hoshino R, Chatani Y, Yamori T, Tsuruo T, Oka H, Yoshida O, Shimada Y, Ari-i S, Wada H, Fujimoto J, et al: Constitutive activation of the 41-/43-kDa mitogen-activated protein kinase signaling pathway in human tumors. Oncogene 18: 813-822, 1999.

35. Amundadottir LT and Leder P: Signal transduction pathways activated and required for mammary carcinogenesis in response to specific oncogenes. Oncogene 16: 737-746, 1998.

36. Alessi DR, Cuenda A, Cohen P, Dudley DT and Saltiel AR: PD 098059 is a specific inhibitor of the activation of mitogenactivated protein kinase kinase in vitro and in vivo. J Biol Chem 270: 27489-27494, 1995. 